\title{
Acute Megakaryoblastic Leukemia with CBFA2T3-GLIS2
}

National Cancer Institute

\section{Source}

National Cancer Institute. Acute Megakaryoblastic Leukemia with CBFA2T3-GLIS2. NCI Thesaurus. Code C132109.

A non-Down syndrome acute megakaryoblastic leukemia that occurs in childhood. It is associated with CBFA2T3-GLIS2 chimeric oncogene and has an unfavorable prognosis. 